\title{
A PROPÓSITO DE LOS GRAFFITI DEL TEMPLO DE SAN MIGUEL DE ESCALADA (LEÓN) ${ }^{1}$
}

\author{
Vanessa JIMENO GUERRA \\ Universidad de León
}

\begin{abstract}
RESUMEN: Aunque los primeros estudios se remontan al siglo XVIII, el templo leonés de San Miguel de Escalada continúa siendo una inagotable fuente de conocimiento. El reciente descubrimiento de sus graffiti parietales nos ha permitido ampliar la información que poseemos sobre este antiguo cenobio, además de establecer una relación, no sólo con otros edificios históricos peninsulares, sino también con la arquitectura que durante la Alta Edad Media fue excavada en sus inmediaciones.
\end{abstract}

PALABRAS CLAVE: San Miguel de Escalada; Graffiti; Alta Edad Media; Arquitectura excavada.

ABSTRACT: In spite of the first studies date back to the 18th century, the leonese temple of San Miguel de Escalada is still being an inexhaustible source of knowledge. Thanks to the recent discovery of its parietal graffiti we have increased the information about this ancient monastery, as well as establish a relation, not only with other historical peninsular buildings, but also with the architecture carved during the High Middle Ages in its surrounding area.

KEYWORDS: San Miguel de Escalada; Graffiti; High Middle Ages; Rock cut architecture.

Desde que en el siglo XVIII el Padre Risco transcribiera la inscripción fundacional del templo de San Miguel de Escalada $^{2}$, han sido muchos los investigadores que, hasta la actualidad, han dirigido sus miradas hacia este antiguo cenobio, aproximándose mediante importantes y cuantiosos estudios a sus testimonios arquitectónicos, artísticos, documentales, epigráficos, así como a su datación cronológica ${ }^{3}$.

${ }^{1}$ Recibido el 5 de julio de 2011. Aceptado en el consejo de 8 de noviembre de 2011.

2 Vid. M. Risco. (1786). España Sagrada. Memorias de la santa iglesia esenta de León, concernientes a los siglos XI, XII y XIII, XXXV, Madrid: Oficina de Pedro Marín, p. 311

${ }^{3}$ Para no realizar una relación excesivamente numerosa, destacamos: J. BAUTISTA LÁzARo. (1903). «San Miguel de Escalada», Boletín de la Sociedad Española de Excursiones, $\mathrm{n}^{\circ}$ 119,120, 121 y 122, pp. 8-11, 36-39, 59-62 y 74-76; F. FiTA. (1897). «San Miguel de Escalada. Inscripciones y Documentos», Boletín de la Real Academia de la Historia, no 31, pp. 466-515; V. GARCÍA LoBO. (1982). Las inscripciones de San Miguel de Escalada: estudio crítico, Barcelona: El Albir, e ID. (2000). Colección documental del monasterio de San Miguel de Escalada, León: Centro de Estudios e 
Pero existen otros elementos dentro de este conjunto que no han tenido igual fortuna a la hora de llamar la atención de los especialistas por ser conceptuados habitualmente como expresiones de carácter marginal. Nos referimos a los graffiti, manifestaciones parietales que, frecuentemente, están presentes en los muros de numerosas arquitecturas históricas tanto dentro como fuera del territorio hispano ${ }^{4}$. Considerados representaciones espontaneas de la sociedad de una época, como bien queda demostrado a través de su diversidad de emplazamientos y motivos, constituyen, en cambio, una importante fuente de conocimiento para la misma ${ }^{5}$. Sin embargo, el hecho de haber sido relegados a un plano más que secundario dentro de las investigaciones científicas, ha desembocado en la ausencia de un necesario corpus que permita establecer análisis comparativos entre los motivos

Investigación "San Isidoro"; H. LARRÉN IZQUIERDO. (2004). «Aspectos visigodos de San Miguel de Escalada (León)», Antigüedad y Cristianismo, n 21, pp. 501-512; J. DE LA FuENTE CRESPO. (2004). «Regestos de documentos de San Miguel de Escalada insertos en el fondo del Monasterio de Trianos», en Escritos dedicados a José María Fernández Catón, vol. 1, León, pp. 504-544; A. MARTíneZ TEJERA. (2004). «Los epígrafes (fundacional y de restauración) del templum de San Miguel de Escalada (prov. de León)», en Congreso Internacional «La Catedral de León en la Edad Media», pp. 613-621; D. ANEDDA. (2004). «La desaparecida inscripción de consagración de la iglesia de San Miguel de Escalada: Un acercamiento prudente», Antigüedad y Cristianismo, no 21, pp. 375388; J. F. ESTEBAN LORENTE. (2005). «La metrología y sus consecuencias en las iglesias de la Alta Edad Media Española. I», Artigrama, pp. 215-254; A. MARTíneZ TeJERA. (2005). El templo del Monasterium de San Miguel de Escalada: “arquitectura de fusión” en el Reino de León (siglos X-XI), Madrid, e I. BANGO TORVISO. (2008). «Los expolios del paisaje monumental y la arquitectura hispana de los siglos VII al XI. Reflexiones sobre el proceso constructivo de San Miguel de Escalada», De Arte, $\mathrm{n}^{\circ}$ 7, pp. 7-50, entre otros.

${ }^{4}$ Algunos de los trabajos que se han ocupado de ellos son, C. NAVARro PovedA. (1991). «Los graffiti medievales del Castillo de la Mola», Betania, no 39, pp. 35-43; L. MARTínez ÁnGEL. (1998). «Sobre los grafitos altomedievales de San Millán de Suso», Estudios Humanísticos. Geografía, Historia y Arte, $\mathrm{n}^{\circ}$ 20, pp. 341-346; C. TEDESCHI. (2000). «Graffiti altomedievali del Tempietto sul Clitunno. A proposito della recente edizione de Carola Jäggi», Scrittura e Civiltà, n²4, pp. 413-419; A. GonZÁlez CoRdero y S. GiLOTTE. (2002). «Graffiti murales de época histórica en el Castillo de Monsalud (Nogales, Badajoz)», Arqueología y Territorio Medieval, nº 9, pp. 249-288; P. OzCÁRIZ GIL. (2007/2008). «Los grafitos del claustro de la Catedral de Pamplona: dibujos destacados y torres medievales», Trabajos de Arqueología de Navarra, ${ }^{\circ}$ 20, pp. 285-310 o V. PRITCHARD. (1967). English Medieval Graffiti, Cambridge: Cambridge University Press.

5 En este sentido, Patrice Cressier considera que los graffiti, "más que cualquier expresión artística oficial", nos ofrecen "información sobre el hombre mismo, sobre la vida cotidiana generalmente ignorada por otras fuentes (tanto escritas como orales). Dicha información es dada tanto por lo que representan los grabados como por las condiciones de su realización (época, lugar) o elección de los temas (motivaciones posibles, etc.)". P. CRESSIER. (1992). «Graffiti cristianos sobre monumentos musulmanes de la Andalucía oriental: Una forma de exorcismo popular», en Estudios de Arqueología Medieval en Almería, Almería, p. 123. 
representados y sus características, así como entre los soportes de los mismos, con el fin de facilitar la compleja labor de identificación y adscripción cronológica ${ }^{6}$.

El descubrimiento de las incisiones parietales practicadas en el templo de Escalada se produce a partir del estudio que hemos realizado sobre los conjuntos arquitectónicos altomedievales que fueron excavados en el valle medio del Esla, el mismo en el que se emplaza el anterior ${ }^{7}$. Los numerosos graffiti que hallamos en el interior de estos espacios y las semejanzas que presentaban con respecto a aquellos conservados en otras arquitecturas peninsulares, tanto excavadas como construidas, durante dicho periodo, nos llevó a emprender una búsqueda de ejemplos similares en los edificios históricos de su entorno más inmediato ${ }^{8}$. Afortunadamente, la investigación efectuada en el templo de Escalada nos ha proporcionado importantes resultados, no sólo para completar este trabajo previo, sino también para aportar una información suplementaria que contribuya a un mejor conocimiento de la historia de este antiguo cenobio leonés y para arrojar luz sobre un aspecto tan desconocido como valioso.

\section{LOCALIZACIÓN Y TIPOLOGÍAS DE LOS GRAFFITI}

Que en el templo de San Miguel de Escalada existían graffiti era algo evidente a tenor de los visiblemente practicados en la arquivolta del vano de acceso al mismo, aunque por esta misma razón resulta incompresible que hayan sido ignorados durante tanto tiempo. Sin embargo, la localización de los restantes ha requerido de una minuciosa y detallada observación de los paramentos y elementos estructurales tanto del interior como del exterior del edificio. Además, ha sido indispensable el

${ }^{6}$ Así, al menos, también lo manifiesta Milagros Guardia en relación a los graffiti hallados en la también leonesa iglesia de Santiago de Peñalba. M. GuARDiA PONS. (2008). «Estudio de los grafitos aparecidos en la iglesia berciana de Santiago de Peñalba», Patrimonio Histórico de Castilla y León, $\mathrm{n}^{\circ}$ 33, p. 58.

${ }^{7}$ V. JIMENO GUERRA. (2010). Arquitectura excavada altomedieval en el valle medio del Esla. (Memoria de Licenciatura inédita). Departamento de Patrimonio Artístico y Documental, Facultad de Filosofía y letras. Universidad de León, León.

${ }^{8} \mathrm{Al}$ igual que otros investigadores, creemos "necesario ampliar los estudios de comparación entre paneles de graffiti sobre soporte histórico y lugares con grabados rupestres de similar cronología, en especial en aquellas áreas donde existan las dos manifestaciones coexistiendo en el tiempo y en el territorio. Dichos estudios comparativos nos permitirán contextualizar temática y cronológicamente muchos yacimientos de grabados rupestres supuestamente hasta ahora esquemáticos, a la vez que nos permitirá contrastar las relaciones reales entre dichas expresiones”. J. I. ROYO GUILLÉN y F. GóMEZ LECUMBERRI. (2002). «Panorama general de los graffiti murales y de los grabados al aire libre medievales y postmedievales en Aragón: Paralelos y divergencias», $\overline{A l}$-Qannī̌s, nº 9, p. 150. 
uso de fuentes de luz artificial de diferente intensidad y ubicadas desde distintos ángulos para que fueran más visibles las incisiones allí practicadas ${ }^{9}$.

A partir de esta intensa labor, los resultados que hemos obtenido nos permiten establecer una mayor concentración de graffiti en dos partes del templo bien diferenciadas: el pórtico sur de acceso, especialmente a lo largo de la superficie de los fustes de las columnas y del vano de entrada, y la nave central, concretamente en las enjutas del segundo arco formero más próximo al presbiterio por el lado norte.

En base a su morfología y temática, hemos creado diversos grupos con el objeto de facilitar el estudio, inventariado e identificación de los mismos, y que a continuación detallamos.

\section{Motivos geométricos}

Este conjunto es el más numeroso de todos ellos, no sólo por la cantidad, sino también por la heterogeneidad de los motivos representados. Por ello, hemos realizado una subdivisión a partir de las formas que éstos presentan.

\section{A. Estructuras reticuladas}

Dentro de esta denominación hemos reunido aquellos graffiti de carácter cuadrangular cuyo interior se encuentra macizado mediante un entramado de líneas horizontales, verticales y oblicuas, que adoptan distintas direcciones dando lugar a un motivo reticular (Ilustraciones 1, 10 y 11).

A pesar de que su significación, si la tuviera, es totalmente desconocida, las estructuras reticuladas son una representación muy frecuente en los espacios cristianos de época altomedieval, destacando, en mayor medida, en aquellos que fueron excavados ${ }^{10}$. El ejemplo más cercano al templo de Escalada se encuentra, en número de dos, en el conjunto de cuevas de la localidad de Villacontilde ${ }^{11}$.

${ }^{9}$ Me gustaría agradecer la ayuda prestada al descubrimiento de los graffiti al Dr. D. Joaquín García Nistal, miembro del Departamento de Patrimonio Artístico y Documental de la Universidad de León, y a Dña. Maria Luisa Giganto.

${ }^{10}$ A. AzKÁRATe Garai-Olaún. (1988). Arqueología cristiana de la Antigüedad Tardía en Álava, Guipúzcoa y Vizcaya, Vitoria-Gasteiz: Diputación Foral de Álava, pp. 461-465; L. A. MonREAL JIMENO. (1989). Eremitorios rupestres altomedievales (El alto valle del Ebro), Bilbao: Universidad de Deusto, p. 111 y A. AzKáRATE Garai-Olaun y I. GarCia CAMINO. (1996). Estelas e inscripciones medievales del País Vasco. (Siglos VI-XI). I. País Vasco occidental, Bilbao: Universidad del País Vasco, p. 105. Igualmente, algunas oquedades naturales que fueron ocupadas durante el periodo medieval conservan en sus muros este tipo de manifestaciones. Como ejemplo de ello véase, J. 
En otras ocasiones, este motivo reticulado no es utilizado en estas estructuras de forma aislada, sino como elemento de relleno de una representación de carácter epigráfico o figurativo. Así, en Escalada observamos la presencia de dos letras "M" a continuación de las cuales se practicó una letra "I" cuyo interior fue aprovechado para realizar este diseño. De la misma manera, son varias las letras " $D$ " que presentan esta particularidad.

Pero no sólo son comunes dentro de las grafías, sino también en otras representaciones de carácter figurativo, cuyos ejemplos los encontramos de nuevo en las inmediaciones del antiguo monasterio, concretamente en las paredes de la denominada «Cueva del Cubo» excavada en la localidad de Rueda del Almirante. En ella fueron practicadas una serie de motivos cruciformes cuyo interior se macizó con estas retículas ${ }^{12}$.

\section{B. Cruciformes}

Las cruces, en todas sus tipologías, y por su evidente carácter simbólico, son uno de los graffiti más habituales en los espacios cristianos, además de por su facilidad de ejecución ${ }^{13}$. En el caso de San Miguel de Escalada suman un total de diecisiete los cruciformes hallados, los cuales pertenecen a dos únicas variantes: cruz latina y cruz patada (Il. 2).

El más sobresaliente de todos ellos es el practicado en la quinta columna del pórtico desde el extremo oriental (Il. 2.2.). Se trata de una evidente cruz de brazos patados cuya peculiaridad reside en el hecho de encontrarse asentada sobre un pedestal, lo que la convierte en una cruz de carácter procesional. Este último ha sido realizado como si de una de las estructuras verticales anteriormente señaladas se tratara, aunque, en este caso, su relleno posee un marcado carácter fitomorfo.

Jiménez Gadea, M. Díaz-Andreu Gracía y T. Pérez Gómez. (2003). «Los grabados medievales del Covacho de las Pintas (Carrascosa de la Sierra, Cuenca)», en I Congrés Internacional de Gravats Rupestres i Murals, Lérida, p. 696.

${ }^{11}$ V. Jimeno GuerRA, Op. cit., pp. 120-122.

12 Algunos de las estructuras verticales y letras macizadas reticularmente que fueron incisas en el interior del templo de Escalada ya han sido puestas en relación con los cruciformes que existieron en esta cueva. V. Jimeno GuERRA, «De pleitos, comisiones y eruditos: la historia de la «Cueva del Cubo» de Rueda del Almirante (León)», De Arte (en prensa).

13 "Dentro de los motivos de carácter religioso, la figura que aparece con mayor profusión son los cruciformes en sus múltiples variantes: desde los motivos más simples de cruces griegas o latinas, con o sin peana y con extremos simples o compuestos, hasta las cruces compuestas, como las patriarcales, las de Caravaca, los calvarios, las cruces con sudario, así como sus derivaciones". J. I. ROYo GuILLÉN y F. Gómez LeCuMBerRi, Op. cit., p. 64. 
Aunque sus características le convierten en uno de los grafitos de mejor ejecución, algunas cruces procesionales semejantes fueron practicadas en el territorio peninsular, como en los muros del monasterio de San Millán de Suso (La Rioja $^{14}$ y en la iglesia de San Pedro de la Rúa (Navarra) ${ }^{15}$.

\section{Esteliformes}

Las representaciones de estrellas de cinco puntas o pentalfas son muy abundantes tanto en el interior como en el exterior del templo, tanto de forma aislada como formando grupos (Il. 3).

Se trata del tipo de esteliforme más representado desde la Antigüedad, por lo que su dilatada presencia en el tiempo y su gran carga simbólica hacen de él un motivo universal de difícil interpretación y adscripción cronológica ${ }^{16}$. No obstante, existe constancia de una desaparición progresiva del mismo entre los siglos XIV y $\mathrm{XV}$, al menos en otros ámbitos peninsulares como el catalán ${ }^{17}$.

Algunas construcciones medievales hispanas como el castillo de Petrer en Alicante o el ya mencionado monasterio de San Millán de Suso son buenos exponentes de la abundante ejecución de este tipo de graffiti $^{18}$.

Igualmente, dentro de esta categoría también incluimos el graffito que hallamos en el intradós del arco formero situado entre el ábside central y el perteneciente a la nave del lado norte. Su morfología de estrella de ocho puntas responde a la combinación de dos cuadrados superpuestos, en ángulo de $45^{\circ}$, de vértices redondeados (Il. 4).

14 M. IbÁÑEz RodrígueZ y T. LeJarRÁGA Nieto. (1998). Los grafitos del Monasterio de San Millán de Suso, Logroño: Consejería de Educación, Juventud, Cultura y Deportes, pp. 24, 153, 155 y 163.

15 P. OzCÁRIZ GiL. (2008). «Nuevos grafitos de San Pedro de la Rúa (Estella) y la ermita de La Almuza (Sesma)», Cuadernos de Arqueología de la Universidad de Navarra, $\mathrm{n}^{\circ}$ 16, pp. 182-183.

16 Así al menos también lo piensan algunos autores como A. CASANOVAS I ROMEU y J. RoviRA I PORT. (2003). «"Status quaestionis" de les representacions gravades medievals a Catalunya. Una visió de conjunt», en I Congrés Internacional de Gravats Rupestres i Murals, Lérida, pp. 663-664 y 666 o C. Navarro Poveda. (2003). «Grafitos medievales del castillo de Petrer y del casttillo de la Mola (Novelda) (Valle Medio del Vinalopó-Alicante)», en I Congrés Internacional de Gravats Rupestres $i$ Murals, Lérida, p. 742.

${ }^{17}$ A. CASAnOvas i Romeu y J. Rovira I PORT. (2003). «Els graffiti i els gravats postmedievals de Catalunya», en I Congrés Internacional de Gravats Rupestres i Murals, Lérida, p. 809.

18 Véase, C. NAVARRo PovedA, Op. cit., pp. 742 y 749 y M. IBÁÑ̃EZ RodríGUEZ y T. LEJARRÁGA NIETO, Op. cit., pp. 138 y 167-169, entre otros. 
En el monasterio de San Millán de Suso se conserva un graffito similar cuya única diferencia con respecto al de Escalada reside en el hecho de que en su interior se practicó una pentalfa. Los investigadores que se encargaron del estudio de este motivo establecieron que se trataba de un signo paleográfico muy habitual en los documentos medievales que se colocaba junto a la rúbrica para dotar la validez $^{19}$. Sin embargo, y a pesar de las semejanzas manifiestas entre estos dos motivos geométricos, creemos que en el caso de San Miguel de Escalada su origen puede encontrarse en la derivación de un elemento decorativo frecuente en las armaduras de cubierta de la Baja Edad Media como es la estrella de ocho puntas.

La gran altura a la que este motivo parietal se encuentra practicado lo hace totalmente inaccesible, por lo que tan sólo pudo ser realizado en un momento en el que el edificio se encontraba en obras. En este sentido, recordemos que fue durante la primera mitad del siglo XV cuando se colocó la armadura de par e hilera sobre la nave central de Escalada, para lo que fue necesario el uso de algún tipo de andamiaje. Además, el alicer superior de dicha cubierta está decorado por una sucesión de lóbulos de ocho y cartelas alargadas a modo de cinta que se asemejan a este perfil doble del lazo inciso, por lo que este motivo pudo servir como fuente de inspiración para la realización de dicho graffito, cuando no fuera alguno de los pintores que decoraron aquellas obras de carpintería, y que conocían sobradamente el motivo de estrella de ocho, su posible ejecutor ${ }^{20}$.

\section{Circulares}

Los motivos circulares incisos en el templo de Escalada se reducen a dos únicos ejemplos de gran perfección en su trazado, lo que denota el uso de un compás en su realización (Il. 5).

Este tipo de representaciones son muy abundantes en los edificios históricos y, generalmente, se han venido relacionando con la construcción de los mismos ${ }^{21}$. La morfología que poseen algunos de ellos, sus divisiones interiores o el lugar en el que se encuentran ubicados son factores bastante indicativos de su carácter

${ }^{19}$ Ibidem, pp. 27-28.

${ }^{20}$ Para una mayor información sobre la cubierta del templo véase J. GARCía NiSTAL. (2007). La carpintería de armar en la provincia de León (ss. XIV-XVIII), tomo II, (Tesis Doctoral inédita). Departamento de Patrimonio Artístico y Documental, Facultad de Filosofía y letras. Universidad de León, León, pp. 823-826.

${ }^{21}$ Ozcáriz recoge numerosos ejemplos practicados en la iglesia del Monasterio de la Oliva, para los que apunta esta posibilidad. P. OzCÁRIz GIL. (2007). Los grafitos de la iglesia del Monasterio de la Oliva (Navarra), Madrid: Universidad Rey Juan Carlos, p. 29. 
arquitectónico. De la misma manera, en otras ocasiones, la condición ornamental que estos graffiti presentan es mucho mayor, por lo que pueden responder a un ensayo previo de un elemento decorativo a realizar.

En el templo de Escalada, uno de estos graffiti, el practicado sobre el fuste de la sexta columna del pórtico por el lado este, presenta una división interior en cuatro partes iguales a partir de dos líneas perpendiculares entre sí, por lo que podría tratarse de algún estudio de proporciones. Mientras, el otro graffito se encuentra en una de las dovelas del mismo arco en el que se representó la ya analizada estrella de ocho puntas, por lo que su realización podría haberse llevado a cabo en el mismo momento y con una finalidad constructiva o de estudio previo, pues no olvidemos que la tablazón que oculta la hilera tiene motivos circulares para los que fue necesario contar con un compás. Asimismo, su constitución a partir de la representación de tres círculos concéntricos es en todo semejante a la de aquellos que Inocencio Redondo recogió en los tirantes de la cubierta de la iglesia de San Julián de los Prados (Oviedo) ${ }^{22}$.

\section{E. Triangulares}

En la tercera columna del pórtico de acceso al templo por el extremo occidental hemos hallado un motivo geométrico formado a partir de la unión de los vértices de dos triángulos, que algunos estudiosos han denominado como pseudolabris $^{23}$ (Il. 6).

A pesar de tratarse de un tema muy habitual en los graffiti medievales, como podemos observar en aquellos practicados en San Miguel de Gormaz (Soria) ${ }^{24}$, en la iglesia del monasterio cisterciense de la Oliva (Navarra) ${ }^{25}$ e incluso en algunas arquitecturas excavadas, como el conjunto de cuevas de Villacontilde, emplazado en las inmediaciones del templo de Escalada ${ }^{26}$, algunos investigadores han logrado establecer dataciones concretas para algunos de ellos. Así, los hallados en Castellfollit de Ruigregós (Barcelona), han sido adscritos a la segunda mitad del siglo $\mathrm{XIII}^{27}$.

${ }^{22}$ I. REDONDO. (1904). Iglesias primitivas de Asturias, Oviedo, p. 41.

23 A. CASANOvas i Romeu y J. RoviRa I PORT, «"Status quaestionis”...», p. 667

${ }^{24}$ B. ARNÁIZ AlONSO. (2008). «La expresión espontánea: Graffiti en San Miguel de Gormaz», en San Miguel de Gormaz. Plan integral para la recuperación de un edificio histórico, Valladolid, p. 196.

${ }^{25}$ P. OzCÁRIZ GIL, Los grafitos..., p. 113.

${ }^{26}$ V. JIMENO GUERRA, Arquitectura excavada..., p. 122.

27 A. CASAnOvas i ROMEU y J. RoviRA I PORT, «“Status quaestionis”...», p. 667. 


\section{F. Lineales}

Dentro de esta categoría tan sólo hemos incluido aquellos graffiti de marcada linealidad y sin morfología definida (Il. 9). Dos de ellos fueron practicados a ambos lados del intradós del vano de acceso al templo, mientras que para los dos restantes se eligió la segunda columna del pórtico por el lado occidental ${ }^{28}$.

\section{Motivos zoomorfos}

Los motivos zoomorfos representados en el templo de Escalada se corresponden con dos de los animales más habituales en los graffiti parietales: el ave y el caballo $^{29}$ (Il. 8).

Del primero de ellos tan sólo hemos hallado un ejemplo en el fuste de la columna más occidental del pórtico (Il. 8.1). Éste se encuentra representado de forma muy esquemática, reducido a las líneas esenciales que definen el animal en cuestión. Sin embargo, en aquellas partes del cuerpo que poseen un mayor plumaje, como el cuello, el ala o la cola, se practicó un mayor número de incisiones. Esta forma de resolver la representación de un ave es similar a la adoptada en el conjunto de cuevas excavadas en la cercana localidad de Villacontilde, donde el único elemento identificativo de la especie de la misma son sus largas patas de zancuda $^{30}$.

También en Escalada fue representado de forma esquemática un caballo, después de dos intentos fallidos, en el intradós del arco formero más cercano al presbiterio por el lado norte (Il. 8.2). Casi la totalidad del cuerpo del animal se encuentra cubierto con líneas practicadas de forma más o menos paralela con el objeto de insinuar el pelaje del équido, al igual que la cerviz, en la que se manifiesta la característica crin. El elemento más llamativo de esta representación es el hecho de que desde su morro atrompetado surgen unas bridas dirigidas hacia el lugar donde debería colocarse el jinete.

Representaciones de caballos semejantes han sido recogidas de forma numerosa en el Monasterio cisterciense de la Oliva (Navarra) ${ }^{31}$ y en el Castillo de Oroners

${ }^{28}$ En este último caso, entre las líneas recogidas parece distinguirse la forma de un pez representado de forma muy esquemática.

29 J. I. Royo Guillén y F. Gómez LeCumberRi, Op. cit., p. 97 y A. CASANOVAS i Romeu y J. ROVIRA I PORT, «"Status quaestionis”...», pp. 672-673;

${ }^{30}$ V. JiMENo GuERra, Arquitectura excavada ..., p. 182.

${ }^{31}$ P. OzCÁRIz GIL, Los grafitos..., pp. 41-42 y 60-62, entre otras. 
(Ager, Lérida) $)^{32}$, entre otros lugares. Aunque no se ha podido establecer una cronología absoluta para este tipo de graffiti, algunos autores los sitúan en el intervalo de tiempo comprendido entre los siglos XII-XV", sobre todo "en el otoño de la Edad Media con el apogeo de la caballería" ${ }^{34}$.

El grupo de los graffiti zoomorfos del templo de Escalada se completa con la figura de otro caballo practicado en la rosca del vano de acceso al templo (Il. 9.1). La representación de éste difiere de forma considerable de aquél inciso en el interior del edificio a tenor de su realismo, lo cual no sólo indica la presencia de una mano diferente sino también su pertenecía a un periodo cronológico distinto. En todo caso, su realización siempre debemos establecerla con posterioridad a 1047-1059, momento en el que el abad Sabarico realizaba este arco en la fachada meridional del templo ${ }^{35}$.

\section{Motivos antropomorfos}

Los dos únicas figuras humanas que hemos hallado se localizan en el pórtico de entrada al templo. La primera de ellas fue incisa en la rosca del arco del vano de acceso al mismo, bajo el caballo anteriormente analizado (II. 9.1). Todas las líneas que la conforman han sido repasadas por la tinta de un bolígrafo como fruto de un injustificado acto vandálico. Su cuerpo se encuentra oculto tras una túnica, dejando tan sólo al descubierto sus pies y brazos, elevados con las manos abiertas. Por la disposición de estos últimos, así como por el atuendo que porta, nos atrevemos a identificarla con la imagen de un orante, tema muy frecuente en los primeros siglos del cristianismo y que perdurará durante toda la Edad Media en algunos países como Italia, Irlanda, España o Francia ${ }^{36}$.

Además de esta imagen, sobradamente conocida por los asiduos visitantes del templo de Escalada, hemos hallado una segunda en el fuste de la columna más occidental del pórtico (Il. 9.2). Se trata de una figura masculina cuyo único elemento significativo y de mayor trascendencia para el análisis de la misma es su calzado puntiagudo. Este tipo de zapato, denominado borceguí, fue utilizado en Europa desde el siglo XIII hasta finales del XV y de él existieron numerosos

\footnotetext{
${ }^{32}$ P. BERTRAN i RoIGÉ y F. Fité i LleVOT. (1984). «Primera aproximació a la ceràmica grisa i als «graffiti» del Castell d’Oroners», Acta Historica et Archaeologica Mediaevalia, n 5-6, p. 407.

${ }^{33}$ P. OzCÁrIz GIL, «Nuevos grafitos...», p. 193.

${ }^{34}$ B. ARNÁIz AlONSO, Op. cit., p. 206.

35 I. BANGO TORVISO, Op. cit., p. 30.

${ }^{36}$ F. CABROL y H. LECLERCQ. (1907-1953). Dictionnaire d'archélogie Chrétienne et de liturgie, vol. XII-II, París: Letouzey et Ané, p. 2292.
} 
modelos, ya que la longitud de las puntas del mismo dependía de la importancia del individuo que los portaba ${ }^{37}$. De esta manera, "los de un príncipe tenían puntas de más de dos pies de largo; los de un barón de dos pies, y de pie y medio los de un simple caballero; las puntas de los zapatos de la gente vulgar tenían medio pie solamente" ${ }^{38}$. Un buen testimonio gráfico de ello son muchas de las miniaturas contenidas en los manuscritos bajomedievales conservados, como las Crónicas de Hainaut, el Roman de la Rose o la Epistre Othea de Christine de Pizan ${ }^{39}$.

No obstante, este tipo de zapato ha sido representado en graffiti medievales localizados en otros ámbitos extra peninsulares como la iglesia británica de Saint Peter en Stetchworth (Cambridgeshire), demostrando así la universalidad de esta $\operatorname{moda}^{40}$.

\section{Motivos epigráficos}

Todos los graffiti de carácter epigráfico que hemos hallado en el templo de Escalada se encuentran en su interior, y más particularmente en las enjutas del segundo arco formero más próximo al presbiterio por el lado norte, a excepción de alguna letra suelta diseminada por los fustes de las columnas del pórtico (Ils. $10 \mathrm{y}$ $3)$.

La lectura de los motivos integrados por letras capitales no reviste gran complicación gracias al tamaño de las grafías, la claridad de las incisiones y la ausencia de erosión, a lo que, a buen seguro, ha contribuído la inaccesibilidad de su emplazamiento. Así, distinguimos el nombre del arcángel al cual se encuentra consagrado el edificio, "MICAEL", y los nombres de algunos de los monjes que pudieron formar parte del antiguo cenobio, "MONIONI MNO" y "PPS MONIONI MONACHO FRUCTO:SO MONACHO"41. Este último conjunto epigráfico presenta como particularidad el hecho de haber sido trazado sobre unas líneas que

${ }^{37}$ J. Ma. MARCHESI. (1849). Catálogo de la Real Armería, Madrid: Aguado, pp. 98-99.

${ }^{38}$ Ibidem, p. 99.

${ }^{39}$ Muchas de las ilustraciones de estos manuscritos se encuentran recogidas en M. DESMOND y P. SHEINGORN. (2003). Myth, Montage, \& Visuality in Late Medieval Manuscript Culture, Michigan: The University of Michigan Press.

${ }^{40}$ En este caso, la figura representada, además de portar borceguíes, aparece ataviada con un vestido francés del siglo XIII. Véase, V. PRITCHARD, Op. cit., p. 60.

${ }^{41}$ El nombre de Monioni aparece formando parte de los testigos de un documento de permuta realizada en el año 940 con el abad Recesvindo de San Miguel de Escalada y los miembros de esta comunidad. Véase, F. FitA Colomé, «San Miguel de Escalada. Inscripciones y documentos», Boletín de la Real Academia de la Historia, $\mathrm{n}^{\circ}$ 31, 1897, pp. 471-472 y, posteriormente, Ed. V. GARCíA LoBO, Colección Documental del Monasterio de San Miguel de Escalada (940-1605), León, 2000, p. 375. 
pretendían actuar a modo de pautado como si de una inscripción se tratase. Además, sobre él se pueden distinguir los trazos de algunos caracteres que, posiblemente, formaban parte de otro conjunto y que fueron ocultados posteriormente bajo el encalado ${ }^{42}$.

Las características de algunas de las grafías que forman parte de estos graffiti epigráficos nos revelan una más que posible escritura visigótica, como es la ausencia de travesaño horizontal en la letra "A", la presencia de un bucle en la parte superior izquierda de la " $T$ " o la forma de "V" que adopta la letra " $U$ "43.

En cuanto a la ubicación de éstos, la única posibilidad que se nos antoja posible para su realización es que se llevasen a cabo en el momento en el que se estaba construyendo el templo, utilizando así algún tipo de plataforma que permitiera acceder a las enjutas de este arco o bien cuando el sillar aún se encontraba a pie de obra. De la misma manera, este procedimiento sería extrapolable a los cruciformes, esteliformes y estructuras reticuladas practicadas en sus inmediaciones, lo cual también permitiría establecer una idéntica cronología para todos ellos.

\section{Signos sin identificar}

Como viene siendo habitual en el estudio de los graffiti históricos, algunos de los motivos representados no poseen una morfología o características definidas que permitan adscribirlos a una categoría determinada. Este es el caso de aquellos hallados en el intradós del arco formero más cercano al presbiterio por el lado sur del templo de Escalada (Il. 12). Se trata de dos elementos aislados macizados con un entramado de líneas que en nada se asemeja a las estructuras reticulares analizadas en el inicio de este estudio. Sin embargo, junto al segundo de los motivos aparece representado lo que se podría identificar con una letra "D".

\section{CONCLUSIONES}

El conjunto de los graffiti practicados en el templo de Escalada presenta una amplia variedad de motivos, pertenecientes, en su mayoría, a los siglos medievales. No obstante, la atemporalidad de muchos de ellos, tales como cruces o estrellas, complica considerablemente su interpretación y datación precisa. Es por ello que, en algunos casos, la cercanía de otros graffiti que sí pueden ofrecer una cronología

\footnotetext{
${ }^{42}$ La costumbre de encalar los muros de los edificios durante el siglo XVII ha provocado que muchos graffiti quedasen ocultos. Un ejemplo de ello son algunos de los practicados en el monasterio de San Millán de Suso. M. IbáÑEz RodríGueZ y T. LejarRáGa Nieto, Op. cit., p. 33.

${ }^{43}$ Un graffiti epigráfico altomedieval practicado en el Monasterio de San Millán de la Cogolla posee estas mismas características, como así lo recoge L. MARTínEZ ÁNGEL, Op. cit., p. 342.
} 
más o menos concreta, como son los de carácter epigráfico, nos ha permitido adscribirlos al mismo periodo e incluso hacerlo extensible a aquellos semejantes practicados en otras partes del templo. Sin embargo, en ocasiones, y a pesar de que los motivos representados pertenecen a la misma categoría temática, las disimilitudes estilísticas o las diferentes características y grosor de sus incisiones son notables, por lo que deben ser contextualizados dentro de un periodo histórico distinto, como sucede en el caso de los équidos.

En otros casos, la inaccesibilidad del emplazamiento de algunos de ellos es el elemento indicativo de que su realización tan sólo fue posible en un momento concreto de la historia del edificio, lo que favorece en gran medida su adscripción cronológica. En este sentido, el conocimiento de las distintas fases constructivas del templo nos proporciona, al menos, una datación post quem para determinados elementos.

La mayor parte de los graffiti que hemos descubierto en Escalada no responden a temas desconocidos por los investigadores, ya que encuentran sus paralelos en algunos edificios peninsulares de carácter religioso y civil. De la misma manera, la arquitectura que fue excavada en su entorno más inmediato también es partícipe de similares manifestaciones parietales, lo que nos permite poner de manifiesto la coexistencia de motivos análogos en espacios creados durante el periodo altomedieval en el mismo contexto geográfico, social y cultural.

A pesar de todo, la importancia de estas manifestaciones no han tenido un reflejo en la atención prestada por los investigadores, motivo por el cual este estudio ha intentado contribuir a la elaboración de ese corpus de graffiti tan demandado por parte de la comunidad científica como necesario, y que constituiría se antoja fundamental como herramienta para el conocimiento, no sólo de las mentalidades, sino también de la diversidad, evolución e iconografía de algunas imágenes. 
(Izda.) Il. 1. Estructura reticulada en una columna del pórtico de acceso al templo de San Miguel de Escalada. Dibujo: autora (Dcha) Il. 2. Graffiti cruciformes practicados em el templo de San Miguel de Escalada (León). Dibujo: autora.
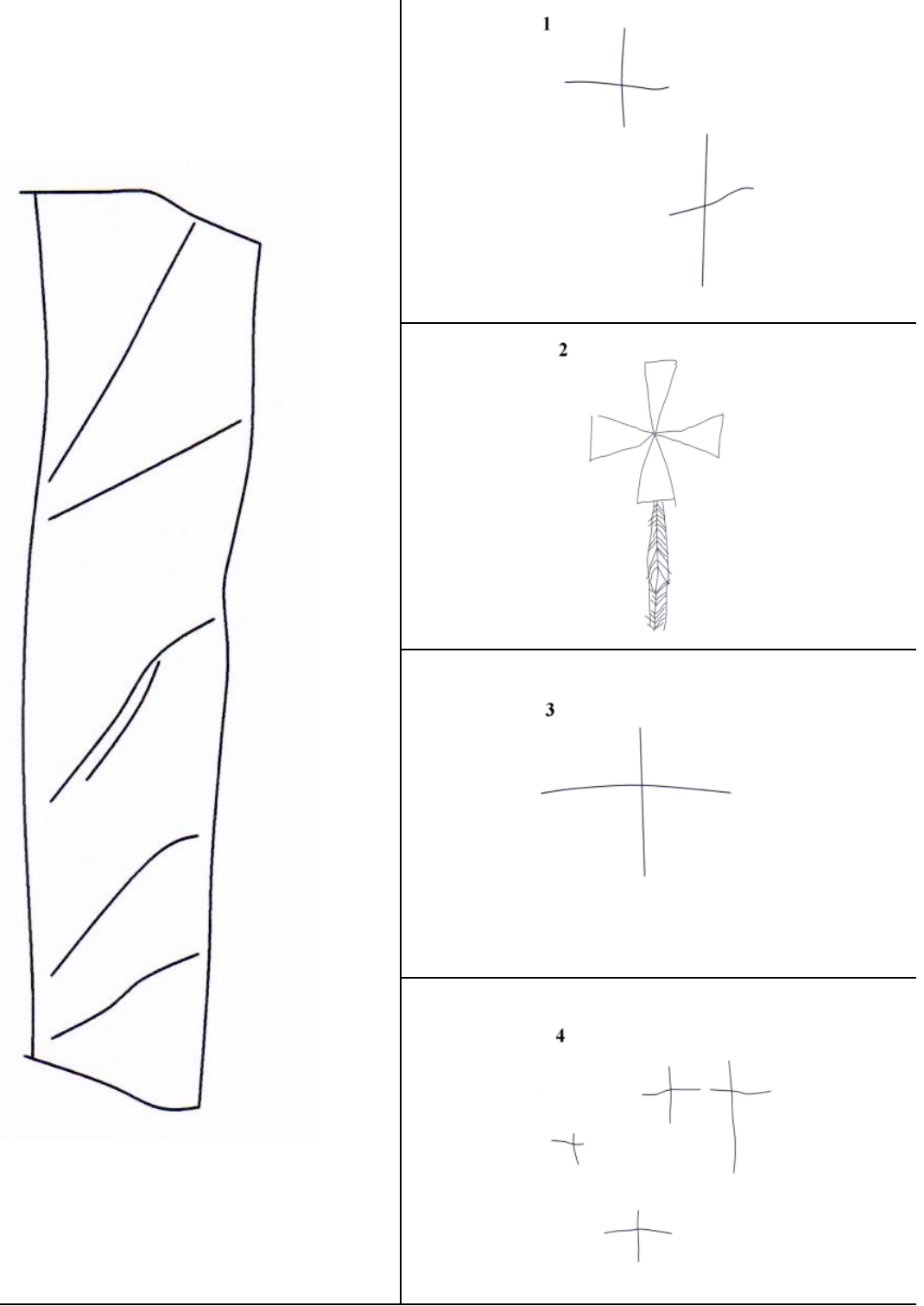

Il. 3. Graffiti esteliformes en el templo de Escalada. Dibujo: 
A propósito de los graffiti del templo de San Miguel de Escalada (León)

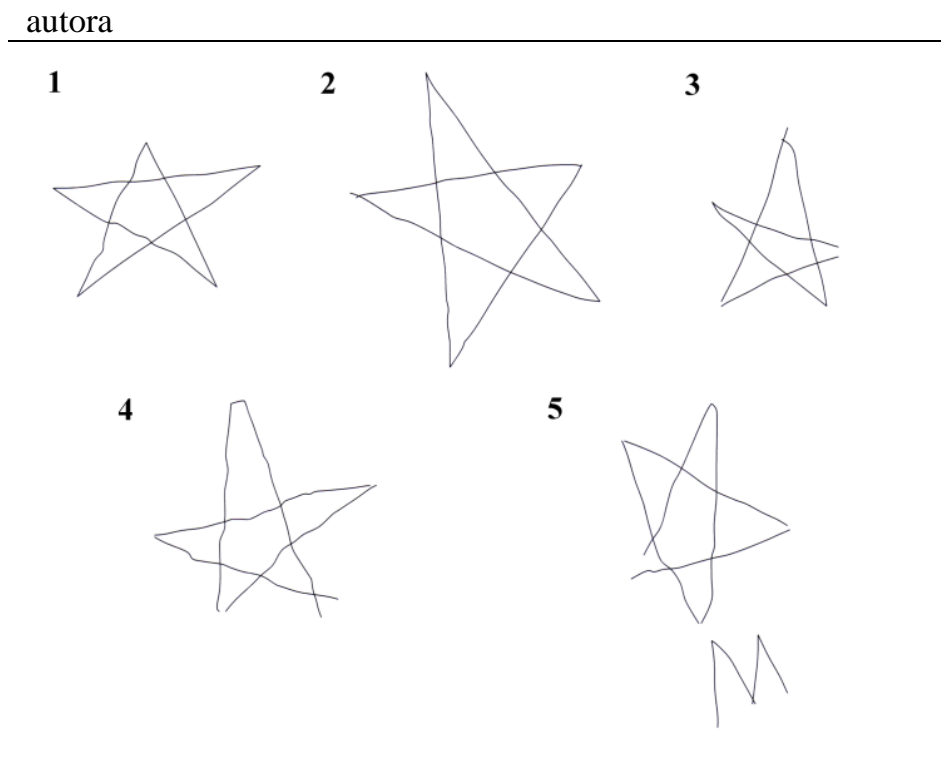

Il. 4. Estrella de ocho puntas con extremos achaflanados en el templo de San Miguel de Escalada (León). Dibujo: autora

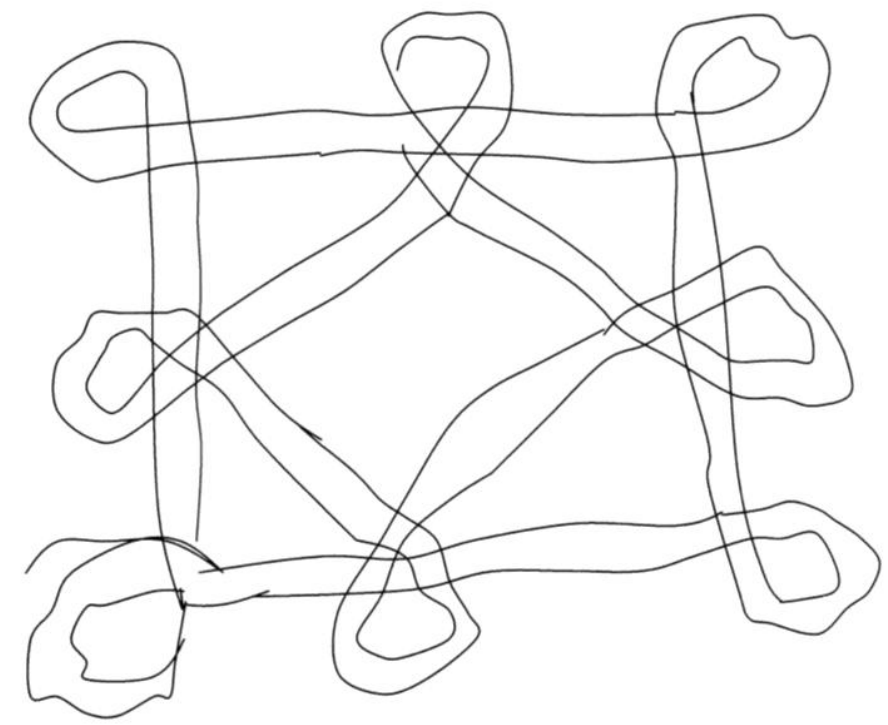


Il. 5. Graffiti circulares en San Miguel de Escalada (León). Dibujo: autora
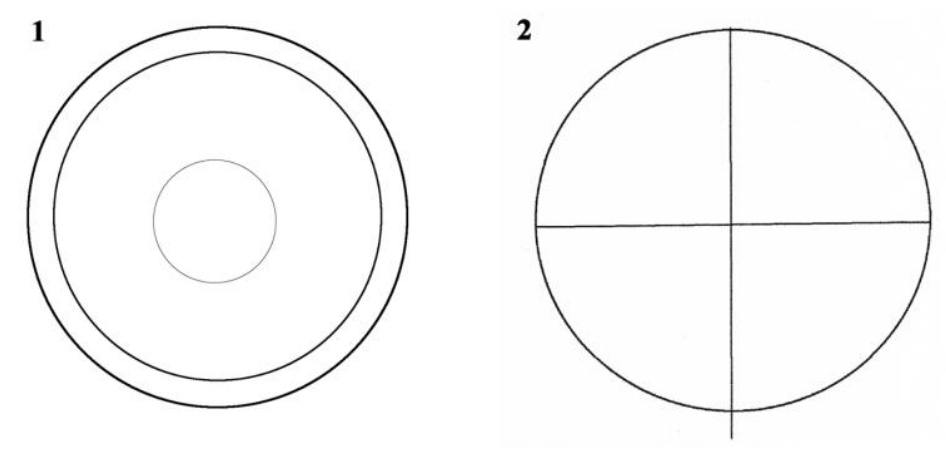

Il. 6. Grafito triangular en el pórtico de acceso al templo de Escalada (León). Dibujo: autora

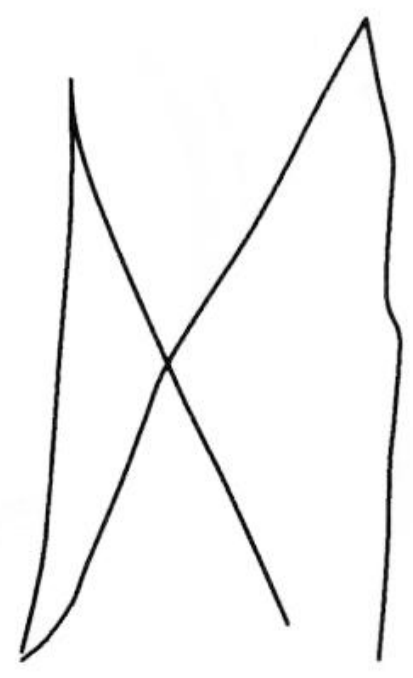


Il. 7. Graffiti lineales en San Miguel de Escalada (León). Dibujo: autora

\section{1}

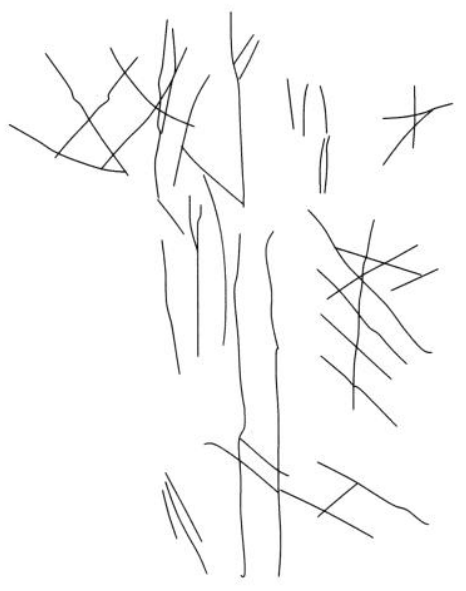

3

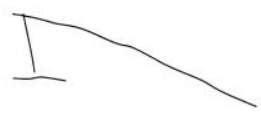

2
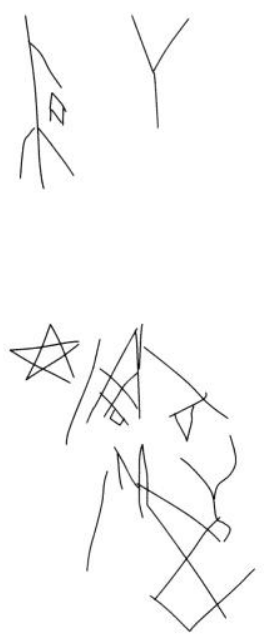

4

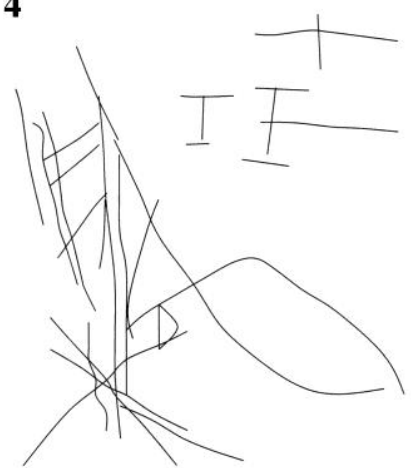


Il. 8. Graffiti antropomorfos en San Miguel de Escalada (León). Dibujo: autora

1

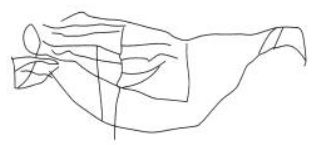

2

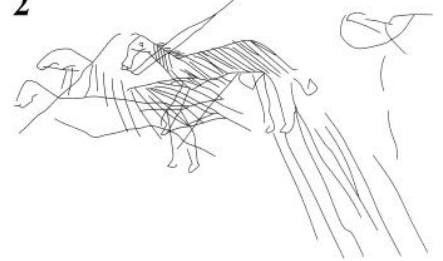

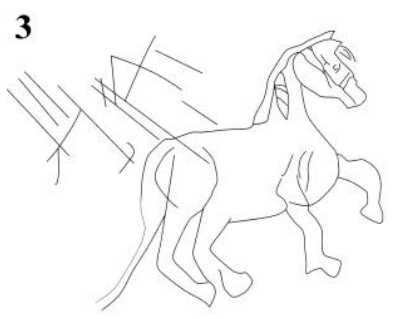

Il. 9. Graffiti antropomorfos en San Miguel de Escalada (León). Dibujo: autora

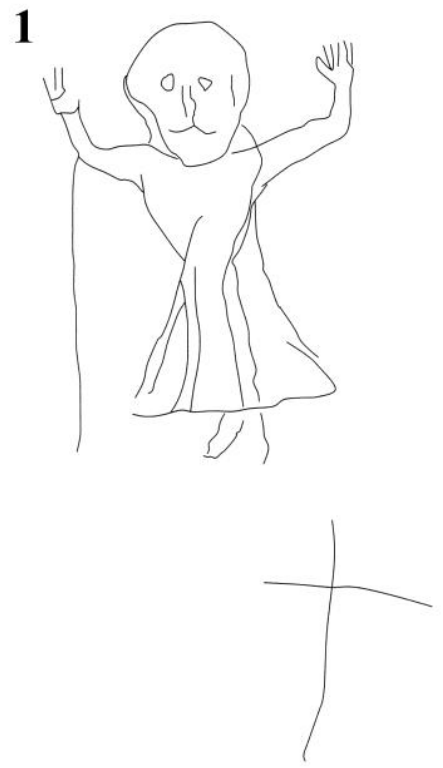

2

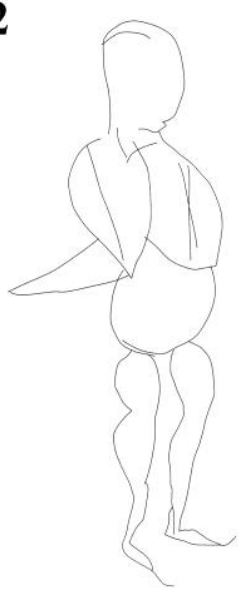


Il. 10. Graffiti en la enjuta oriental del arco formero más cercano al presbiterio de la iglesia de San Miguel de Escalada (León). Dibujo: autora.

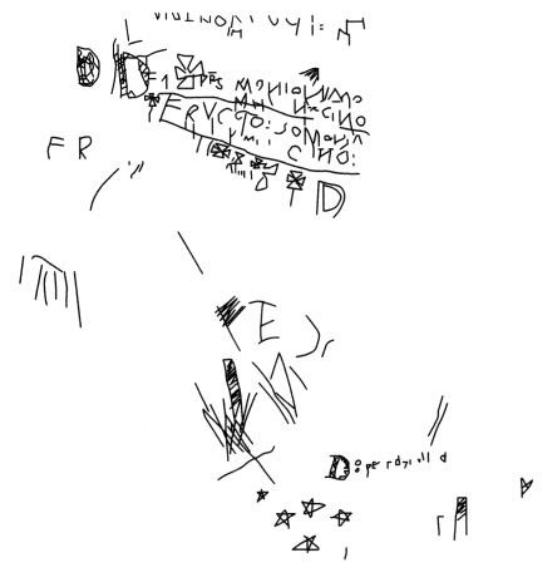

Il. 11. Graffiti en la enjuta occidental del arco formero más cercano al presbiterio. Iglesia de San Miguel de Escalada (León). Dibujo: autora

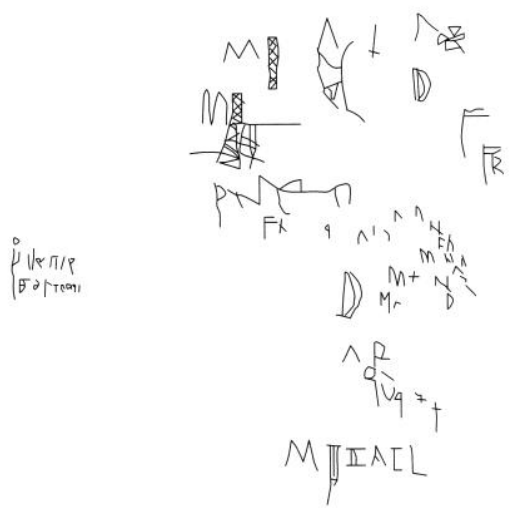


Il. 12. Graffiti sin identificar en el interior de la Iglesia de San Miguel de Escalada (León). Dibujo: autora
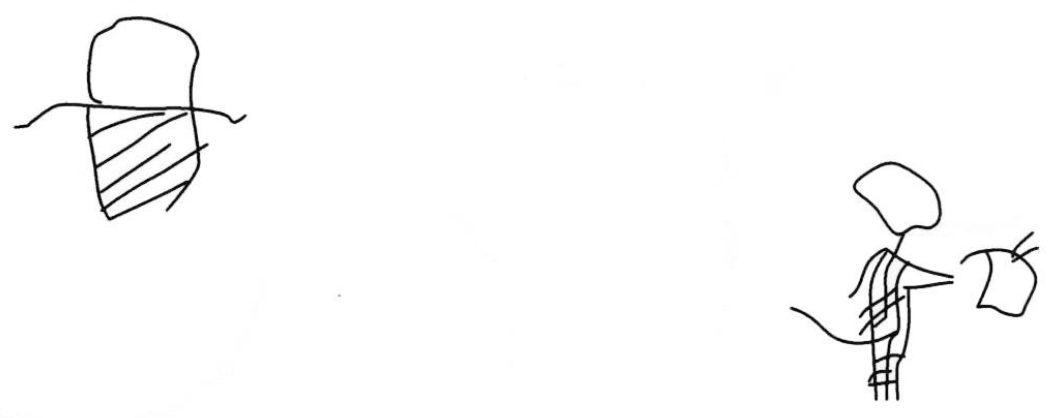